Do regional gender and racial biases predict

gender and racial biases in social face judgments?

(PSA001 Secondary Analysis Challenge)

\author{
DongWon Oh \\ New York University
}

Alexander Todorov

Princeton University

Corresponding author:

DongWon Oh

6 Washington Place

New York, NY 10003

orcid.org/0000-0002-2105-3756

Email: dongwon.oh@nyu.edu 


\begin{abstract}
Trait impressions from faces are more simplified for women than men. This bias stems from gender stereotypes; when strong stereotypes exist for a group of faces (e.g., of women's or Blacks'), they are evaluated more positively/negatively when they fit/violate the stereotypes, making the impressions simpler (i.e., more one-dimensional). In this preregistered study, using trait impression ratings of faces collected from various world regions $(+11,000$ participants in 48 countries), scores of implicit associations $(+18,000$ and $+212,000$ participants in +200 countries $)$, and mixed-effects models, we ask (1) whether simplified facial impressions are found for women and Blacks across regions and (2) whether the regional level of stereotypes about genders and races is correlated with the level of simplification in the face-based impressions of women and Blacks, respectively. The results were not coherent across analyses. The interpretation of the results and the limitations of the study are discussed.
\end{abstract}




\section{Do regional gender and racial biases predict gender and racial biases in social face judgments?}

People form simplified facial impressions of women; face-based trait judgments of women are more strongly intercorrelated than those of men (Oh, Dotsch, Porter, \& Todorov, 2019; Sutherland, Young, Mootz, \& Oldmeadow, 2015). Separate statistical modeling of male and female faces suggests that the bias stems from stereotype-induced evaluation after initial face perception (Oh et al., 2019) - the degree to which a face belonging a group fits (or violates) the group stereotype. For instance, when a male face appears competitive (or gentle), the face is evaluated positively (or negatively), whereas when a female face appears competitive (or gentle), the face is evaluated negatively (or positively). Prior evidence suggests that to the extent that individual perceivers endorse gender stereotypes, they form more simplified impressions of faces (Oh et al., 2019). Based on these findings, the authors argued that "beyond the differences in impressions of male and female faces", the cross-group difference in impression intercorrelations might extend to "other face subcategories in which one or more subcategories are more stereotyped or prejudiced against than the others, such as race."

In the current preregistered study, by examining regional measures of gender and race biases, we test whether face-based trait attributions of female and non-white faces (i.e., faces with potentially stronger expectations about personality traits than white or male faces) may be more simplified (i.e., more highly intercorrelated) than those of male and white faces, respectively. In addition to the average trend between groups (e.g., men and women; white and Black faces), the level of bias should be correlated with the level of stereotype endorsement in individuals living in each region, in line with previous work (Oh et al., 2019). 
Regional measures reflecting group biases, such as the regional mean scores of Implicit Association Test (IAT; Greenwald, McGhee, \& Schwartz, 1998), have been shown to predict meaningful social outcomes at the regional level. For instance, children's science and math achievement in school was predicted by the regional gender prejudice IAT (Nosek et al., 2009), and the police's use of lethal force was predicted by the regional racial prejudice IAT score (Hehman, Flake, \& Calanchini, 2018). The validity of these measures has been established (Hehman, Calanchini, Flake, \& Leitner, 2019). Here, following the previous regional analyses, we predict the level of bias that exist in face-based trait judgments from the regional level of the social-group related bias, defined by the index of gender and racial prejudice.

\section{Method}

All materials, including the preregistered analysis script and bias measures, are available at https://osf.io/erpvm.

Participants. We used face-image ratings collected from over 11,000 participants in 48 countries (Jones et al., 2020). The ratings were collected for Psychological Science Accelerator-001 (PSA001). PSA-001 was a world-wide multi-laboratory collaboration testing the replicability of the classic finding of the $2 \mathrm{~d}$ structure in face evaluation (valence $\times$ physical power; Oosterhof $\&$ Todorov, 2008) across various world regions. The PSA-001 dataset allowed us to quantitatively assess the level of gender and racial face biases in these regions, and thus was suitable for testing our hypotheses.

Stimuli and Procedure. Participants were asked to evaluate Asian, Black, Latino, and white faces from a standardized database (Chicago Face Database; Ma, Correll, \& Wittenbrink, 2015), one face at a time. Faces were presented on a computer screen in a random order. Instructions were given in each country's official language. Each participant rated all faces on a single randomly 
selected dimension out of 13 trait dimensions (aggressive, attractive, caring, confident, dominant, emotionally stable, intelligent, mean, responsible, sociable, trustworthy, unhappy, weird). See Jones et al. (2020) for details of data properties and the procedure of data acquisition. Regional bias measures. For regional bias measures, we used Project Implicit Demo Website datasets (Nosek et al., 2018), specifically, gender and race prejudice IAT scores, collected from $+18,000$ and $+212,000$ participants respectively in +200 countries in 2018 alone. For gender bias, country-level indices represented gender inequality, country- and state-level means IAT score representing the associations of "Men=Career" and "Women=Family". For racial bias, countryand state-level means IAT score representing the associations of "white=Good" and "Black=Bad".

For gender bias, as a complimentary measure to the gender prejudice IAT score, a country-level gender-bias index (Country Gender Bias Index, henceforth) was computed and used. The Country Gender Bias Index was defined as the first component score of countries derived from a principal component analysis (PCA) solution of six country-level indices related to gender inequality. Of the six indices, three were calculated by global organizations: 2018 Global Gender Gap Index (World Economic Forum, 2018), 2018 Gender Inequality Index (United Nation Developoment Programme, 2018), and 2017 Gender Development Index (United Nation Developoment Programme, 2019). For each index, we used the most recent year's index publicly available as of November 2019 (i.e., the time of preregistration). The other three indices were objective values indicative of gender equality for each country: the gender ratio of parliament/congress seats in 2017, the gender ratio of secondary education, and the gender ratio of labor force. The resulting Country Gender Bias Index (a unitary value for each country) explained $53.80 \%$ of the variance in all six indices. 
Analytic approach. Using gender and racial bias measures at the level of countries and US-states, we predict the level of facial-judgment simplicity. The level of facial-judgment simplicity was defined by two measures. The first facial bias measure was the variance in face-based judgments explained by the first Principal Component of the judgment ratings (in line with Oh et al. 2018). The second facial bias measure was the correlation between face-based judgments of trustworthiness and dominance (in line with Sutherland et al., 2015). These two measures were calculated for each race face subcategory (white vs. Black faces) and gender face subcategory (male vs. female faces). We employed mixed-effects regression models to consider the hierarchical structure of the data (multiple face categories (male/female or white/Black) nested in every region). We expected that non-maleness (and non-whiteness) of faces, as well as stronger regional biases, would be associated with more simplified (i.e., highly intercorrelated) face-based trait judgments (fixed effects). Variables that may potentially confound the effect were included in our models as covariates; for example, \% male IAT participants in each country can potentially increase the biasing effect in the face ratings because men tend to show higher gender-IAT scores (that is, a stronger association between men with career and women with family) than do women because men tend to be more gender-prejudiced than women are. As such, following previous regional analyses (e.g., Nosek et al., 2009), we included covariates to consider potential confounds in each regression model whenever relevant, to minimize the potential confounding effects. We included $\%$ male IAT participants and $\%$ male PSA face raters in each region (i.e., country or state) in all gender-bias analyses involving IAT scores; and \% male PSA face raters in each country in the gender-bias analysis involving gender-inequality index. The GDP of each country was additionally included for all country-level analyses because per-country GDP should be correlated with the country-level gender and racial equality. Due to 
the similarity in the structure and variables adopted across many models, we set alpha at 0.01 to minimize Type I Errors.

\section{Results}

Gender-bias analysis: Across regions (not preregistered). Before examining the effect of the regional bias, we first tested whether the previously found gender biases in facial impressions replicated in the current dataset. We considered the mean ratings averaged across all regions and races. First, we separately correlated the trustworthiness and dominance ratings of male and female faces (T-D correlation; as in Sutherland et al., 2015). T-D correlation was positive in direction for both male $(n=60 ; r=.19, t(58)=1.54, p=.129)$ and female faces $(n=60 ; r=.36$, $\mathrm{t}(58)=2.95, \mathrm{p}=.005)$, but significant only for female faces. The stronger T-D correction in female ratings is consistent with previous findings (Oh et al., 2019; Sutherland et al., 2015). However, previous research found a negative correlation between trustworthiness and dominance ratings in female faces whereas the current analyses found a positive correlation (see General Discussion for possible interpretations). Second, we separately calculated the variance explained by PC1 in male and female face ratings (as in Oh et al., 2019; Var_PC1; a proxy for valence in face evaluation with traits of a sufficient number and range). Var_PC1 was higher for male (67.59\%) than female faces $(64.86 \%)$. This result was inconsistent with previous research found a higher Var_PC1 for female than male faces across datasets (Oh et al., 2019).

Conducting the same two analyses in each face-race category yielded mixed results (male vs. female Asian faces: r=.01 vs .48 , Var_PC1=58.70 vs. 57.35 ; Black faces: $r=.60$ vs. .40, Var_PC1=79.35 vs. 71.10; Latino faces: -.04 vs. .05, Var_PC1=75.65 vs. 57.34; white faces: $\mathrm{r}=.13$ vs. .39, Var_PC1 $=62.83$ vs. $72.77 ; \mathrm{n}=15$ for each face gender $\times$ race subcategory) with white faces being the only race category showing a cross-gender difference in the direction that 
is consistent with previous findings (i.e., less differentiation in female than male face-based trait judgments) although unexpectedly, the female T-D correlation was positive for white faces.

These results suggest that the basic effect of gender biases in the form of more simplified facial impressions for female faces may be weak or not generalizable across world regions and face sexes.

Race-bias analysis: Across regions (not preregistered). The same two analyses were conducted to test for racial biases (i.e., correlations between trustworthiness and dominance ratings; PCAs). T-D correlation was positive both in white $(\mathrm{n}=120 ; \mathrm{r}=0.24, \mathrm{t}(28)=1.30, \mathrm{p}=.204)$ and Black faces $(\mathrm{n}=120 ; \mathrm{r}=.49, \mathrm{t}(28)=2.96, \mathrm{p}=.006)$ but significant only for Black faces. Var_PC1 was higher for Black (75.95\%) than white faces (70.30\%). Both analyses suggested that Black faces were evaluated in a more simplified fashion than were white faces.

Conducting the same two analyses in each face-sex category yielded mixed results (white vs. Black male faces: $\mathrm{r}=.13$ vs .60 , Var_PC1=62.83 vs. 79.35 ; white vs. Black female faces: $\mathrm{r}=.39$ vs. .40 , Var_PC1 $=72.77$ vs. $71.10 ; \mathrm{n}=30$ for each face gender $\times$ race subcategory) with male faces showing a cross-race difference in the direction that is consistent with the working hypothesis of the present study (i.e., less differentiation in Black than white face-based trait judgments). However, as in gender analyses (described in the previous Results section), unexpectedly, the Black T-D correlation was positive. These results suggest that the effect of race biases in the form of more simplified facial impressions for Black faces may be weak or not generalizable across world regions and face races. Alternatively, this may mean race biases work in a distinct way from gender biases (e.g., when a Black face appears "dominant" it conforms to the social norm (unlike a female face, in which "facial dominance" violates the social norm), 
therefore the person is perceived as positive - trustworthy - resulting in the positive T-D correlation in Black faces).

Gender-bias analysis: Country-level. A mixed-effects model predicting Var_PC1 from the country-level Gender prejudice IAT score (the level of associations of "Women=Home, Men=Career") was conducted. The final dataset had 31 countries with the number of face raters ranging from $n=27$ (Russia) to $n=2,273$ (the US) for each country (mean $n=281.70$, median $n$ $=174$ ), and the number of IAT participants ranging from $n=27$ (Ecuador) to $n=139,226$ (the US) for each country (mean $n=5,487.00$, median $n=328$ ). The model did not find the main effect of the IAT score or the interaction effect between the IAT score and the face sex (Table 1 top).

As a complimentary analysis, a mixed-effects model predicting Var_PC1 from the Country Gender Bias Index was conducted. The final dataset had 29 countries with the number of face raters ranging again from 27 (Russia) to 2,273 (the US) for each country (mean $n=295.70$, median $n=201$ ). The model did not find the main effect of the Country Gender Bias Index or the interaction effect between Country Gender Bias Index and the face sex (Table 1 bottom). No other effects were significant.

Table 1. Fixed effects results of the country-level gender-bias analysis (dependent variable $=\%$ Var explained by PC1).

B SE $\quad$ df $\quad t \quad p$ Analysis based on Gender IAT score

$\begin{array}{llllll}\text { GDP } & 0.336 & 0.170 & 24 & 1.97 & 0.060 \\ \text { \% male face raters } & 0.234 & 0.211 & 24 & 1.11 & 0.279 \\ \text { \% male IAT participants } & 0.022 & 0.170 & 24 & 0.13 & 0.897\end{array}$




\begin{tabular}{lccccc} 
Face sex (male) & 0.006 & 0.071 & 27 & 0.09 & 0.932 \\
Gender IAT & 0.393 & 0.215 & 24.11 & 1.82 & 0.081 \\
Gender IAT × Face sex & 0.025 & 0.072 & 27 & 0.35 & 0.728 \\
\hline & Analysis based on the Country Gender Index & & \\
\hline & 0.747 & 0.253 & 27 & 2.95 & 0.006 \\
GDP & -0.021 & 0.166 & 27 & -0.13 & 0.898 \\
\% male face raters & -0.028 & 0.069 & 29 & -0.40 & 0.689 \\
Face sex (male) & 0.362 & 0.258 & 28 & 1.41 & 0.171 \\
Gender index & -0.063 & 0.069 & 29 & -0.91 & 0.369 \\
Gender index $\times$ Face sex & & & & & \\
\hline
\end{tabular}

A mixed-effects model predicting the level of T-D correlation from the Gender IAT score was conducted. The model did not find the main effect of the IAT score or the interaction effect between the IAT score and the face sex (Table 2 top). A mixed-effects model predicting T-D correlation from the Country Gender Bias Index was conducted. The model did not find the main effect of the Country Gender Bias Index or the interaction effect between Country Gender Bias Index and the face sex (Table 2 bottom). The only significant effect was the effect of the face sex; across the two models considered, ratings of trustworthiness and dominance were more strongly positively correlated for female faces than male faces in the positive direction.

Table 2. Fixed effects results of the country-level gender-bias analysis (dependent variable $=$ Pearson correlational coefficient between trustworthiness and dominance ratings). B SE $\quad$ df $\quad t \quad p$ 


\begin{tabular}{lccccc} 
GDP & 0.186 & 0.164 & 24 & 1.13 & 0.270 \\
\% male face raters & 0.318 & 0.204 & 24 & 1.56 & 0.131 \\
\% male IAT participants & -0.198 & 0.164 & 24 & -1.21 & 0.239 \\
Face sex (male) & -0.734 & 0.160 & 27 & -4.60 & $<0.001$ \\
Gender IAT & 0.214 & 0.223 & 31.14 & 0.96 & 0.344 \\
Gender IAT × Face sex & -0.012 & 0.161 & 27 & -0.07 & 0.941 \\
\hline & Analysis based on the Country Gender Index & & \\
GDP & 0.325 & 0.246 & 27 & 1.32 & 0.196 \\
\% male face raters & 0.060 & 0.161 & 27 & 0.38 & 0.710 \\
Face sex (male) & -0.721 & 0.151 & 29 & -4.78 & $<0.001$ \\
Gender index & 0.150 & 0.254 & 28.79 & 0.59 & 0.561 \\
Gender index $\times$ Face sex & 0.041 & 0.152 & 29 & 0.27 & 0.787 \\
\hline
\end{tabular}

Gender-bias analysis: State-level. A mixed-effects model predicting the variance explained by Var_PC1 from the Gender IAT score was conducted. The final dataset had 16 states with the number of face raters ranging from $n=17$ (Georgia) to $n=497$ (California) for each state (mean $\mathrm{n}=145.80$, median $\mathrm{n}=111$ ). The model did not find any effect involving the Gender IAT score (Table 3). No other effects were significant.

Table 3. Fixed effects results of the state-level gender-bias analysis (dependent variable $=\%$ Var explained by PC1).

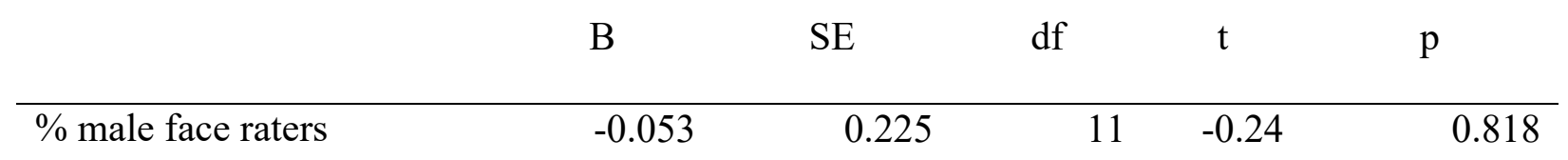




\begin{tabular}{lrrrrr} 
\% male IAT participants & -0.041 & 0.218 & 11 & -0.19 & 0.854 \\
Face sex (male) & 0.227 & 0.128 & 13 & 1.78 & 0.099 \\
Gender IAT & -0.368 & 0.225 & 12.88 & -1.64 & 0.126 \\
Gender IAT $\times$ Face sex & -0.051 & 0.126 & 13 & -0.40 & 0.694 \\
\hline
\end{tabular}

A mixed-effects model predicting T-D correlation from the state-level Gender IAT score was conducted. The model found a significant effect of Gender IAT; the higher the state-level Gender prejudice IAT score was, the more negatively trustworthiness and dominance ratings were correlated to each to other for both male and female faces (Table 4). No other effects were significant.

Table 4. Fixed effects results of the state-level gender-bias analysis (dependent variable $=$ Pearson correlational coefficient between trustworthiness and dominance ratings).

\begin{tabular}{llrrrr} 
& B & \multicolumn{2}{l}{ SE } & df & \multicolumn{2}{c}{$\mathrm{p}$} \\
\hline \% male IAT participants & -0.140 & 0.120 & 10 & -1.17 & 0.268 \\
\% male face raters & 0.174 & 0.121 & 10 & 1.44 & 0.181 \\
Face sex (male) & -0.347 & 0.211 & 12 & -1.65 & 0.125 \\
Gender IAT & -0.834 & 0.217 & 11.22 & -3.84 & 0.003 \\
Gender IAT $\times$ Face sex & 0.211 & 0.203 & 12 & 1.04 & 0.318 \\
\hline
\end{tabular}

Race-bias analysis: Country-level. A mixed-effects model predicting the variance explained by Var_PC1 from the country-level Race prejudice IAT score (the level of associations of "Black=Bad, white=Good") was conducted. The final dataset had 18 countries with the number 
of face raters ranging from $n=46$ (Finland) to $n=2,273$ (the US) for each country (mean $n=378.2$, median $\mathrm{n}=223.5$ ), and the number of IAT participants ranging from $\mathrm{n}=23$ (Ecuador) to $\mathrm{n}=190,660$ (the US) for each country (mean $\mathrm{n}=11,026.22$, median $\mathrm{n}=243$ ). The model did not find the main effect of the IAT score or the interaction effect between the IAT score and the face race (Table 5). The only significant effect was the effect of the face race; Var_PC1 higher for black than white faces.

Table 5. Fixed effects results of the country-level race-bias analysis (dependent variable $=\%$ Var explained by PC1).

\begin{tabular}{llcccc} 
& B & SE & df & t & p \\
\hline GDP & 0.448 & 0.219 & 14 & 2.04 & 0.060 \\
\% white IAT participants & -0.395 & 0.225 & 14 & -1.76 & 0.100 \\
Face race (white) & -0.676 & 0.160 & 16 & -4.22 & 0.001 \\
Race IAT & 0.007 & 0.229 & 18 & 0.03 & 0.976 \\
Race IAT $\times$ Face race & 0.062 & 0.162 & 16 & 0.38 & 0.708 \\
\hline
\end{tabular}

A mixed-effects model predicting T-D correlation from the Race IAT score was conducted. Again, we did not find the main effect of the IAT score or the interaction effect between the IAT score and the face race (Table 6). The only significant effect was the effect of the face race; the correlation between trustworthiness and dominance ratings were higher for black than white faces. 
Table 6. Fixed effects results of the country-level race-bias analysis (dependent variable $=$ Pearson correlational coefficient between trustworthiness and dominance ratings).

\begin{tabular}{llcccc} 
& B & SE & df & t & p \\
\hline GDP & 0.084 & 0.244 & 14 & 0.35 & 0.735 \\
\% white IAT participants & -0.573 & 0.222 & 16 & -2.58 & 0.020 \\
Face race (white) & -0.292 & 0.245 & 14.62 & -1.19 & 0.253 \\
Race IAT & 0.286 & 0.225 & 16 & 1.27 & 0.223 \\
Race IAT $\times$ Face race & 0.084 & 0.244 & 14 & 0.35 & 0.735
\end{tabular}

Race-bias analysis: State-level. A mixed-effects model predicting the variance explained by Var_PC1 from the state-level Race prejudice IAT score (the level of associations of "Black=Bad, white=Good") was conducted. The final dataset had 16 states with the number of face raters ranging from $n=17$ (Georgia) to $n=497$ (California) for each state (mean $n=145.80$, median $\mathrm{n}=111$ ). The model did not find the main effect of the IAT score or the interaction effect between the IAT score and the face race (Table 7). The only significant effect was the effect of the face race; Var_PC1 higher for black than white faces. This is consistent with the country-level racebias analysis.

Table 7. Fixed effects results of the state-level race-bias analysis (dependent variable $=\%$ Var explained by PC1).

\begin{tabular}{llcccc} 
& B & SE & df & t & p \\
& & & & & \\
\hline \% Black IAT participants & -0.265 & 0.287 & 12 & -0.92 & 0.374 \\
\% Black state residents & -0.687 & 0.303 & 12 & -2.27 & 0.043
\end{tabular}




\begin{tabular}{lccccc} 
Face sex (white) & -0.822 & 0.087 & 14 & -9.48 & $<0.001$ \\
Race IAT & 0.035 & 0.221 & 13 & 0.16 & 0.875 \\
Race IAT $\times$ Face sex & 0.086 & 0.088 & 14 & 0.97 & 0.346 \\
\hline
\end{tabular}

A mixed-effects model predicting T-D correlation from the state-level Race IAT score was conducted. Again, we did not find the main effect of the IAT score or the interaction effect between the IAT score and the face race (Table 8). The only significant effect was, again, the effect of the face race; the ratings of trustworthiness and dominance were more strongly correlated for Black faces than white faces in the positive direction.

Table 8. Fixed effects results of the state-level race-bias analysis (dependent variable $=$ Pearson correlational coefficient between trustworthiness and dominance ratings).

\begin{tabular}{llcccc} 
& B & SE & df & t & p \\
\hline \% Black IAT participants & 0.084 & 0.305 & 11 & 0.28 & 0.788 \\
\% Black state residents & -0.494 & 0.353 & 11 & -1.40 & 0.189 \\
Face sex (white) & -0.703 & 0.177 & 13 & -3.96 & 0.002 \\
Race IAT & -0.403 & 0.247 & 14.68 & -1.63 & 0.124 \\
Race IAT $\times$ Face sex & 0.260 & 0.188 & 13 & 1.38 & 0.190 \\
\hline
\end{tabular}


Overall, no clear pattern of statistical significance was observed across analyses. We discuss the interpretations of significant and non-significant effects as well as potential limitations of the current analyses.

Significant face category effect. In non-preregistered analyses, we found that trustworthiness and dominance ratings were more strongly correlated for female than male faces, and for Black than white faces, but the correlation was always in the positive direction. Var_PC1 was not larger for female than male faces. All in all, these results suggest that the effect of gender and race biases in the form of more simplified facial impressions may be weak, or not generalizable across world regions and face subcategories. However, it is noteworthy that the effective sample size (i.e., number of faces) for the replication of the previous effect (i.e., white faces; Oh et al., 2019) here was 120 in the present dataset $(n=15$ per face-gender $\times$ race subcategory), whereas the original study adopting stimuli from the same face database used the sample size of 597 ( $n=\geq 51$ per facegender $\times$ race subcategory; Oh et al., 2019, Study 1c). Depending on the physical characteristics of sample faces, the correlational structure of social judgments can dramatically vary; for example, if a sample of faces happen to be submissive-appearing on average, then more dominance can lead to positive evaluation, resulting in a positive T-D correlation (through more facial typicality; Langlois \& Roggman, 1990; Sofer, Dotsch, Wigboldus, \& Todorov, 2015), whereas a sample of faces happen to be dominant-appearing on average, then more dominance can lead to negative evaluation, resulting in a negative T-D correlation (through more atypicality). Indeed, a unpreregistered, post hoc examination of Chicago Face Database (CDF) norming data (Ma et al. 2015) revealed that in each of all 8 gender $\times$ race subcategories, 15 face samples selected in the current dataset had on average lower dominant ratings than all face stimuli from the entire database (Asian male: $\mathrm{CFD}=2.71$ vs. $\mathrm{PSA}=2.60$; Asian female: $\mathrm{CFD}=2.30$ 
vs. $\mathrm{PSA}=2.26$; Black male: $\mathrm{CFD}=3.43$ vs. $\mathrm{PSA}=3.21$; Black female: $\mathrm{CFD}=2.83$ vs. $\mathrm{PSA}=2.68$;

Latino male: $\mathrm{CFD}=3.21$ vs. $\mathrm{PSA}=3.06$; Latino female: $\mathrm{CFD}=2.55$ vs. $\mathrm{PSA}=2.55$; white male: $\mathrm{CFD}=2.94$ vs. $\mathrm{PSA}=2.89 ; 1-7$ scale from the original $\mathrm{CFD}$ norming data) with the exception of white female faces $(\mathrm{CFD}=2.48$ vs. $\mathrm{PSA}=2.49)$. These values suggest that some of the unexpected results (e.g., the positive T-D correlations) might have been due to the biased representation in the sample (i.e., overall lower dominant ratings in the sample faces employed here).

In preregistered analyses, we found the main effect of face category (i.e., face sex and face race) in some models; in gender-bias analyses, at the country level, ratings of trustworthiness and dominance were more strongly and positively correlated for female faces than male faces. In race-bias analyses, both at the country and state levels, Var_PC1 was higher for black than white faces. At the state level, the ratings of trustworthiness and dominance were more strongly correlated for Black faces than white faces in the positive direction. These significant results, identical in their directions, suggest that trait judgments from non-white male faces (i.e., female and Black faces) do tend to be more simplified than judgments from their "default" counterparts (i.e., male and white faces). Again, as previously described (see the beginning of this section, Significant face category effect), the increased level of intercorrelations in impressions of non-male, non-white faces is consistent with previous predictions (Oh et al., 2019) and empirical findings (Oh et al., 2019; Sutherland et al., 2015). This may mean that female and Black faces were perceived in a more simplified manner than were male and white faces, respectively. However, given the inconsistency in the pattern of statistical significance across analyses (5 null results from 10 analyses), the effect appears weak.

Significant regional bias effect. In preregistered analyses, we found the main effect of the regional bias; at the state level, the higher the state-level Gender prejudice IAT score was, the 
more negatively trustworthiness and dominance ratings were correlated to each to other. The relationship between the level of simplification in facial impressions and the stereotype held by individuals in the perceivers' geographical surroundings is consistent with the prediction and empirical finding of Oh et al. (2019); in this paper, perceivers' gender-stereotype endorsement positively predicted their level of impression simplification. This result may mean that faces were perceived in a more simplified manner when the perceivers are from a state where the residents hold strong gender stereotypes. However, given the null effect of the regional gender bias in other models ( 9 null results from 10 analyses), this effect appears weak.

Interpretation of null results. Overall, we did not find any significant effect of interest in most analyses (face category effect: 5 of 10 analyses; regional bias effect: 9 of 10 analyses). This pattern of results can be interpreted in multiple ways, which are not mutually exclusive from one another.

One possibility is that no expected reliable relationship exists - no relationship between the face categories and impression intercorrelations and no relationship between the impression intercorrelations and the regional level of prejudice. This possibility, however, seems unlikely in light of preceding evidence (Oh et al., 2019; Sutherland et al., 2015) and the significant effects in the current analyses (note that no effect that was significant in the opposite direction of our preregistered predictions).

Another possibility is about the reliability of the variables - both (1) independent and (2) dependent variables. First, the regional IAT scores and Country Gender Bias Index in the current datasets (i.e., independent variables) may not be a reliable measure of the level of prejudice held by individuals living in the corresponding regions. Although a large number of participants across regions took part in gender and race IATs used in the current analyses, many states and 
countries had a small sample (e.g., face-gender analyses: $n=27$ in Ecuador; face-race analyses: $\mathrm{n}=23$ in Ecuador; also note the small medians). As a result, in these areas, the IAT scores might have not reflected the actual level of stereotype/prejudice in the regions. Future research can increase the reliability of the measurements by aggregating the participants across datasets, for example across multiple timepoints (e.g., using IAT scores averaged across multiple past years, rather than using IAT scores collected in 2018 alone, as in the current report). Second, our measurements of facial bias - regional values of Var_PC1 and T-D correlation - (dependent variables) may not reflect the "true" values. Similar to the independent variables' reliability issue, many regions had a small number of raters (e.g., face-gender analyses: $n=27$ in Russia and $n=17$ in Georgia; face-race analyses: $n=46$ in Finland and n=17 in Georgia; also note the small medians). This could have led to an inaccurate estimate of the level of biases in these regions, thereby making our final analyses unreliable. Additionally, as described in the previous section (see Significant face category effect), the sample faces employed here may be biased in their appearances, adding to the unreliability of the dependent variables (i.e., Var_PC1 and T-D correlation).

The issue of small numbers of viable participants suggests another possibility; the last possible explanation of the many null results is that the dataset did not have enough number of regions for our statistical models. Even if we had a small number of individual subjects in the face ratings and IAT scores in some regions (described in the previous paragraph), this shortcoming could have been overcome by a sufficient number of datapoints in the final models (i.e., sufficient observations in our linear mixed-effects models). However, the final datasets had only handful of datapoints (i.e., face-gender analyses: 31 countries (analysis using the IAT score), 29 countries (analysis using Country Gender Bias Index), and 16 states; face-race analyses: 18 
countries and 16 states), underpowering our tests. This was partially because it was hard to find states and countries whose data were available across multiple dataset types (e.g., face-rating data and IAT data, face-rating data and Country Gender Bias Index). Future research can find another regional bias measure that maximizes the utility of the PSA-001 dataset (i.e., a bias measure that has as many countries/states existing in the PSA-001 dataset as possible), or collect and/or employ a new dataset entailing a larger number of regions. 


\section{Reference}

Greenwald, A. G., McGhee, D. E., \& Schwartz, J. L. (1998). Measuring individual differences in implicit cognition: the implicit association test. Journal of Personality and Social Psychology, 74(6), 1464.

Hehman, E., Calanchini, J., Flake, J. K., \& Leitner, J. B. (2019). Establishing construct validity evidence for regional measures of explicit and implicit racial bias. Journal of Experimental Psychology: General, 148(6), 1022-1040. doi:10.1037/xge0000623

Hehman, E., Flake, J. K., \& Calanchini, J. (2018). Disproportionate use of lethal force in policing is associated with regional racial biases of residents. Social Psychological and Personality Science, 9(4), 393-401. doi:10.1177/1948550617711229

Jones, B. C., DeBruine, L. M., Flake, J. K., Liuzza, M. T., Antfolk, J., Arinze, N. C., .. . Coles, N. A. (2020). To which world regions does the valence-dominance model of social perception apply? (Registered Report Stage 2). PsyArXiv.

Langlois, J. H., \& Roggman, L. A. (1990). Attractive faces are only average. Psychological Science, 1(2), 115-121. doi:10.1111/j.1467-9280.1990.tb00079.x

Ma, D. S., Correll, J., \& Wittenbrink, B. (2015). The Chicago face database: A free stimulus set of faces and norming data. Behavior Research Methods, 47(4), 1122-1135.

Nosek, B. A., Smyth, F. L., Sriram, N., Lindner, N. M., Devos, T., Ayala, A., . . Greenwald, A. G. (2009). National differences in gender-science stereotypes predict national sex differences in science and math achievement. Proceedings of the National Academy of Sciences, 106(26), 10593-10597. doi:10.1073/pnas.0809921106 
Nosek, B. A., Sutin, E., Hansen, J. J., Wu, L., Sriram, N., Smyth, F. L., . . Greenwald, A. G. (2018). Project Implicit: Advancing theory and evidence with technical and methodological innovation. PsyArXiv, PsyArXiv.

Oh, D., Dotsch, R., Porter, J., \& Todorov, A. (2019). Gender biases in impressions from faces: Empirical studies and computational models. Journal of Experimental Psychology: General. doi:10.1037/xge0000638

Oosterhof, N. N., \& Todorov, A. (2008). The functional basis of face evaluation. Proceedings of the National Academy of Sciences, 105(32), 11087-11092. doi:10.1073/pnas.0805664105

Sofer, C., Dotsch, R., Wigboldus, D. H. J., \& Todorov, A. (2015). What is typical is good: the influence of face typicality on perceived trustworthiness. Psychological Science, 26(1), 39-47. doi:10.1177/0956797614554955

Sutherland, C. A. M., Young, A. W., Mootz, C. A., \& Oldmeadow, J. A. (2015). Face gender and stereotypicality influence facial trait evaluation: Counter-stereotypical female faces are negatively evaluated. British Journal of Psychology, 106(2), 186-208.

United Nation Developoment Programme. (2018). Human development data (1990-2018). Retrieved from: http://hdr.undp.org/en/data

United Nation Developoment Programme. (2019). Gender Development Index. Retrieved from: http://hdr.undp.org/sites/default/files/hdro_statistical_data_table4.pdf

World Economic Forum. (2018). The global gender gap report. 